\title{
Quantification of Structural Correlation in STEM Images using the Projected Pair Distribution Function
}

\author{
Matthew J. Cabral ${ }^{1}$, Elizabeth C. Dickey ${ }^{1}$ and James M. LeBeau ${ }^{1}$ \\ 1. Department of Materials Science \& Engineering, North Carolina State University, Raleigh, NC.
}

Picometer-scale structural distortion drives a wide range of material properties including pyroelectricity, ferroelectricity, and piezoelectricity. Further, since short-range correlations of chemistry and distortion in these systems manifest unique properties, it is crucial to quantify and understand how they contribute to material response. In this presentation, we will discuss application of the projected pair distribution function (pPDF) for identifying short-range structural correlation in STEM images. The pPDF, which is a 2D analogue of the PDF technique employed in diffraction, provides a representation of the distances between neighboring atom column pairs directly from STEM images as illustrated in Figure 1a [1]. The pPDF can be separated into partial pPDFs to investigate the distance distribution between constituent atom column types in an image [2].

As an example application, we will apply the analysis to $\mathrm{Pb}\left(\mathrm{Mg}_{1 / 3} \mathrm{Nb}_{2 / 3}\right) \mathrm{O}_{3}(\mathrm{PMN})$, a prototypical relaxor with the perovskite structure where the A sub-lattice (composed of $\mathrm{Pb}$ ) and the $\mathrm{B}$ sub-lattice (composed of $\mathrm{Mg} / \mathrm{Nb} / \mathrm{O}$ ) can be readily separated into the pPDFs for each sub-lattice as in Figure $1 \mathrm{~b}$. As evident from the pPDFs, the A sub-lattice of PMN has a significantly broader distribution than the B sub-lattice indicating structural distortion in the Pb-containing atom columns. The distortion for each sub-lattice can be further investigated by analyzing the standard deviation, $\sigma$, of the pPDF peaks as a function of distance. Figure 1c illustrates the magnitude of $\sigma$ oscillates for the A sub-lattice while remaining consistent for the B sub-lattice. For reference, the pPDF for a Si single crystal illustrates that for a crystal without correlated distortions, the standard deviation remains flat [3].

We will discuss how modeling of distortions within a periodic lattice is used to determine the origin of trends in the pPDFs. In each of these models $2 \mathrm{~nm}$ nanodomains are randomly distributed on otherwise perfect lattice. In the case of isotropic volumetric strain, the $\sigma$ vs. distance plot (Figure $2 a$ ) illustrates an alternating trend in the width of the pPDF peaks. Additional structural distortions are also presented (Figures 2b-c) incorporating both shear and volumetric strain. As evident from the modelled pPDFs, varying types of structural distortion result in different significantly different $\sigma$ profiles. We will demonstrate the effect of each type of distortion on the pPDF for a variety of domain configurations and length scales to determine the factors contributing to the pPDF of structurally complex materials such as PMN. Finally, we will discuss the broad applicability of this technique and how the pPDF can be used to identify the type and length scale of structural distortion in STEM images with varying structure [4]. 


\section{References:}

[1] X. Sang et al, Appl. Phys. Lett. 106(6) (2015).

[2] X. Sang, A. A. Oni, and J. M. LeBeau, Microsc. Microanal 20 (2014), p. 1764.

[3] J. H. Dycus et al, Microsc. Microanal. July (2015), p. 1.

[4] This material is based upon work supported by the National Science Foundation, as part of the Center for Dielectrics and Piezoelectrics under Grant Nos. IIP-1361571 and IIP-1361503. The authors acknowledge the use of the Analytical Instrumentation Facility at NCSU, which is supported by the state of North Carolina and the National Science Foundation. M.J.C acknowledges support from the National Science Foundation, as part of NRT-SEAS under Grant No, DGE-1633587.
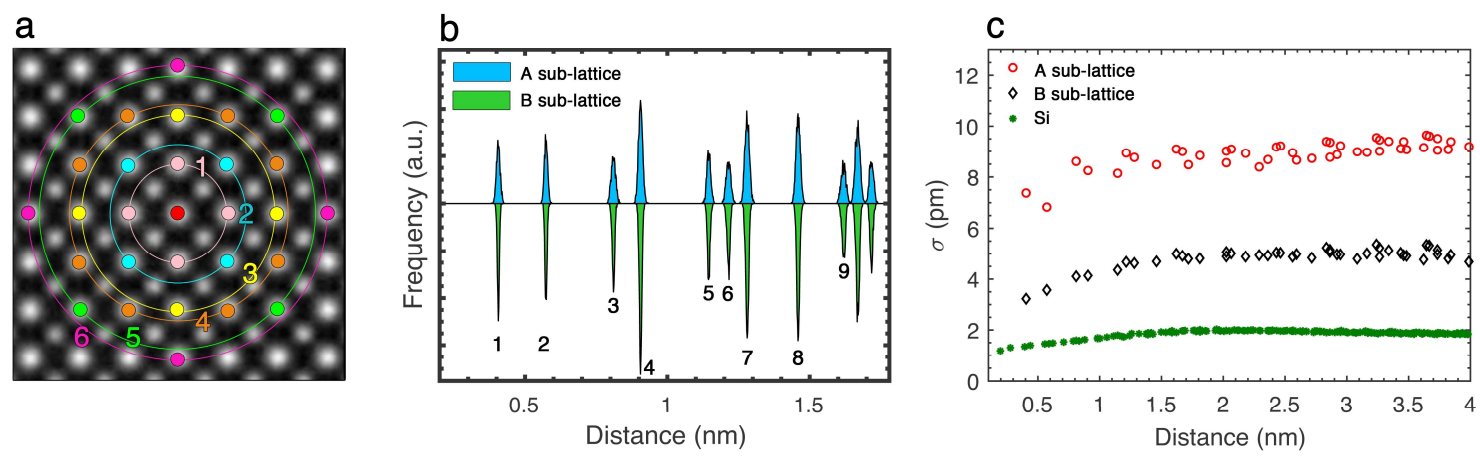

Figure 1. (a) Schematic of the projected pair distribution function (pPDF). (b) Partial pPDFs for the A (blue) and B (green) sub-lattice positions in PMN. (c) Standard deviation, $\sigma$, of the pPDF distribution as a function of distance for the A (red) and B (black) sub-lattice positions. The pPDF for Si is represented in green to illustrate a flat pPDF for a cubic material.
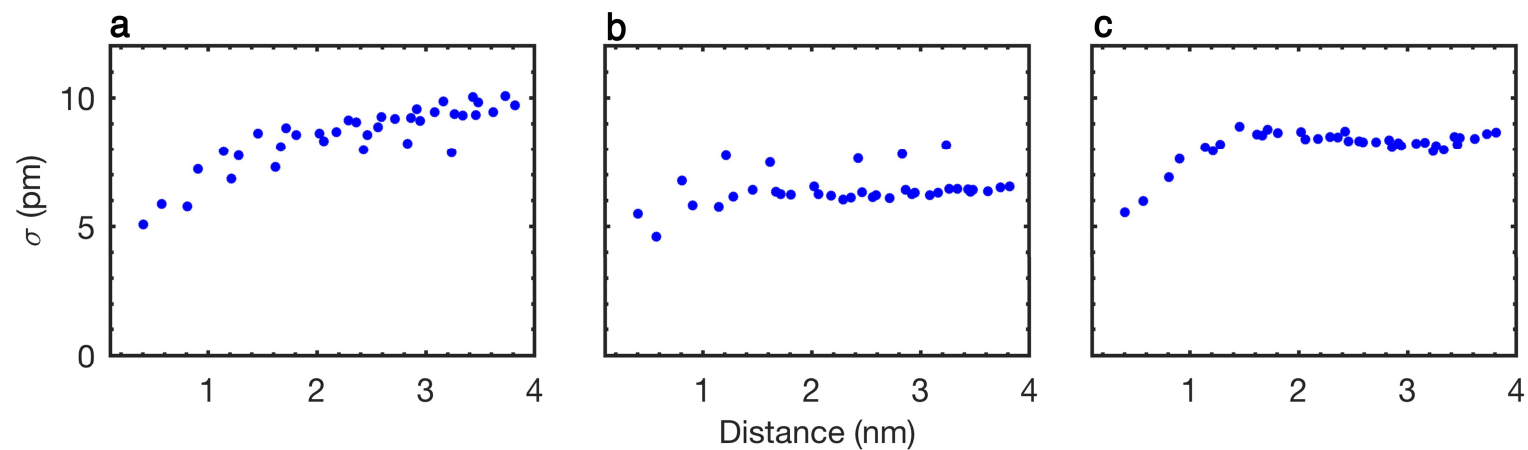

Figure 2. PPDF $\sigma$ vs. distance distribution for randomly distributed nanodomains with (a) 3\% isotropic strain (b) 3\% shear and (c) strained and sheared structures. 